\title{
Instrumentation for the California Extremely Large Telescope
}

Keith Taylor, Ian S. McLean

Keith Taylor, Ian S. McLean, "Instrumentation for the California Extremely Large Telescope," Proc. SPIE 4841, Instrument Design and Performance for Optical/Infrared Ground-based Telescopes, (7 March 2003); doi: $10.1117 / 12.461782$

SPIE Event: Astronomical Telescopes and Instrumentation, 2002, Waikoloa, Hawai'i, United States 


\title{
Instrumentation for the California Extremely Large Telescope
}

\author{
Keith Taylor ${ }^{a}$ and Ian McLean ${ }^{b}$ \\ ${ }^{a}$ Astronomy Dept., California Institute of Technology, CA 91125 \\ ${ }^{b}$ Department of Physics and Astronomy, UCLA, CA 90095
}

\begin{abstract}
The Phase A study for the California Extremely Large Telescope (CELT) Project ${ }^{1}$ has recently been completed. As part of this exercise a working group was set-up to evolve instrumentation strategies matched to the scientific case for the CELT facility. We report here on the proposed initial instrument suite which includes not only massively multiplexed seeing-limited multi-object spectroscopy but also on plans for wide-field adaptive optics fed integral-field spectroscopy and imaging at, or approaching, CELT's diffraction limit.
\end{abstract}

Keywords: CELT; Extremely Large Telescopes ; Instrumentation ; Integral Field Units

\section{INTRODUCTION}

In order to take full advantage of an increase in telescope aperture (A), instruments need to scale proportionately to maintain their information gathering capacity (or $A \Omega$ ). The size scaling of instruments to telescopes is only relaxed when a decrease in sky aperture $(\Omega)$ is afforded through finer atmospheric seeing conditions aided where possible by adaptive correction of the incoming wavefront. The development of the 8-metre telescopes coincided with the realization that to fully capaitalize on their investment improvement in the atmospheric quality of the chosen sites coupled with the development of adative optics (AO) was essential. Consequently some mitigation in the otherwise proportionate increase in instrument size was obtained. Unfortunately for CELT there are no obvious indications of significant improvements in atmospheric conditions beyond that characteristic of Mauna Kea or the good Chilean sites and hence a return to a simple scaling law for instrument size is a reasonable, if daunting, first order conclusion.

Compounding this problem is the fact that, while telescope apertures gains have been dwarfed in comparison to the gains afforded through the development of detectors, the detector pixel-size has not scaled with telescope aperture. Pixels are generally at $\sim 20 \mu \mathrm{m}$ (or below) while, with the development of edge-butting, quasi-contiguous formats can in principle grow to any linear size. For Nyquist sampling, camera f-ratios scale inversely with telescope aperture hence seeing limited $(>0.5 ")$ apertures require impossibly fast (f/0.4) f-ratios, while field angles, rather than detector formats, become the limiting factor in information capacity.

Of course, such considerations beg the question of what type of information is to be recorded - spectral, spatial, temporal or a combination of all three. Pure imaging will have a limited but important role in the near- and mid-IR however there is little doubt that spectroscopy will dominate in all wavebands. Filling the available detector real estate is then generally a choice between pure cross-dispersed high-resolution echelle spectroscopy, multi-object spectroscopy (MOS) at a more limited spectral resolution or some hybrid of the two. A recent addition to this more traditional arsenal is the development of so called " $3 \mathrm{D}$ " or "tri-dimensional" spectroscopy most commonly integral field units (IFUs) and tunable narrow-band filters (eg: Fabry-Perots or Imaging Fourier Transform Spectrographs). Taking the CELT science case ${ }^{1}$ as our lead, we conclude that classical MOS techniques are favored for optical, seeing-limited, spectroscopy while 3D techniques, exemplified in particular by IFUs, are preferred in the multi-conjugate AO-fed, near-IR domain. There is clearly cross-talk between these two broad alternatives - eg: AO-fed near-IR MOS and seeing-limited IFTS work - however we limit our discussion to those techniques which are most evidently in demand. 


\section{SEEING-LIMITED INSTRUMENTS}

The baseline assumption here is that seeing limited instrumentation will be exclusively employed in the optical domain given the impracticability of adaptively correcting the incoming wave-front for $\lambda<1 \mu \mathrm{m}$ over a wide field of view. The demand for maximal throughput UV/optical spectroscopy, unencumbered by the inevitable losses of $\mathrm{AO}$ systems, is expected to remain strong despite the fact that in many cases the necessary use of relatively large spectroscopic apertures will compromise sky-background limited observations even for high dispersion ( $\mathrm{R} \sim 50,000$ ) cross-dispersed echelle spectroscopy. In principle an extremely high-order AO-fed instrument would be far superior however competitive efficiencies would have to be established down into the UV in order to make such a proposal viable and even then such gains would only apply for point sources.

Indeed relatively large spectroscopic apertures ( $>0.5$ ") are already necessary in order to integrate over marginally resolved objects such as distant galaxies ${ }^{2}$. Integral field units, while offering the potential to reduce aperture size and hence sky background flux, are necessarily expensive in detector real-estate and hence in object (or wavelength) multiplex and are most effective on high surface brightness, line emission regions of complex sources. Taking object multiplex into account, faint absorption-line galaxies, marginally resolved in good $(<0.5$ ") seeing, are still more efficiently studied using a classical MOS (multislit or multi-fiber) device ${ }^{3}$.

We are thus left with the demand to investigate ways of catering for classical, cross-dispersed echelle spectroscopy and MOS in the seeing limited optical régime. Any attempt to design spectrographs for seeing limited work on CELT will require very fast cameras - faster than $\mathrm{f} / 1$, if possible. Furthermore, the natural demand to maximize object and/or wavelength multiplex implies pushing camera designs to very large field angles. Fortunately Vogt has demonstrated ${ }^{4}$ a viable design for an $800 \mathrm{~mm}$ beam, $\sim \mathrm{f} / 1.2$ camera operating from $0.3-1 \mu \mathrm{m}$. Given the inevitable cost and size of these cameras our approach is thus to use them as a vast information collection resource that can be used for multiplexed observations in either the spatial or spectral domain. This leads to the MTHR concept for a single object, cross-dispersed, high dispersion echelle combined with a multi-fiber intermediate dispersion spectrograph and associated fiber positioner.

\subsection{Celt High Resolution Spectrograph (CHRS)}

CHRS, like UVES, is a dual-beam instrument consisting of two parallel red and blue arms. Each arm is a grating-cross-dispersed echelle spectrometer in a UVES dual-white-pupil configuration, with a scaled-up HIRES-style camera feeding a CCD array of $8 \mathrm{k}^{2}, 15 \mu \mathrm{m}$ pixels. While the blue and red sides would be coating-optimized the HIRES-style cameras and mirror-collimators are almost totally achromatic and hence both sides can work over much larger wavelength regions (a factor which will prove very useful for fiberfeeding as discussed later). The R-4 echelles are very large by present standards (1.0 by 3.5 -meters in size) and would require a 5-by-8 mosaic of the largest presently available. A grating cross-disperser would be used to diffract the light into a HIRES-style catadioptric camera with a CCD array at its internal focus.

\subsection{Moderate Resolution Multi-Object Fiber-fed Mode (MTHR)}

The large and expensive cameras, gratings, and large CCD mosaics in CHRS can also be used to good advantage for moderate resolution fiber-fed multi-object spectroscopy, providing resolutions in the 2,00020,000 range for hundreds of objects at a time. A HIRES-style camera (used in reverse) serves as a fibercollimator, accepting light at $\mathrm{f} / 4.5$ from the outputs of a line of fibers. The collimated beams pass through two large fused-silica corrector lenses, and then off a grating replacing the CHRS cross-disperser and into the normal CHRS camera. Both arms of CHRS could have such a fiber feed. 
The grating for this multi-fiber mode could be the same grating used for cross-dispersion for the highresolution mode. However that grating would need to be rotated $180^{\circ}$ about its normal to stay on the same side of its blaze. Most probably, other optimized gratings would be used, mounted on a cross-disperser carousel alongside the high resolution cross-disperser.

With fiber systems there is little freedom to customize input aperture size to prevailing atmospheric conditions or to the intrinsic object size and hence we have to establish a suitable compromise. Clearly point source observations call for smaller apertures however previous studies have focused on optimization for distant galaxy work. We now have the tools for refining such a choice however for arguments sake we will adopt an aperture of 0.75 ", somewhat biased for marginally resolved galaxies, as a suitable compromise. Now using the established fiber-IFU technologies, exemplified by the Anglo-Australian Observatory's SPIRAL project ${ }^{5}$, we can invoke a hexagonally formatted fiber-slicer aperture as an input to CHRS. Clearly the finer the slicing the higher the resolution until the $\sim 2$-pixel resolution limit is reached. Here we analyse the trade-off in object multiplex $(\mathrm{M \# )}$ with slicer format assumming a camera f-ratio of $\sim \mathrm{f} / 1.1$, core:clad ratios of 1.1 and a 1-pixel separation between each object spectrum.

If we examine the effect of different hexagonal slicing choices then, as can be seen in Table 1, diminishing returns in $\mathrm{R}$ are encountered beyond the 7-hex arrangement due to the fibers approaching the 2-pixel limit. Indeed if the requirements were heavily biased towards object multiplex rather than spectral resolution then one could use a single, un-sliced fiber aperture, however, with the possibility of a low resolution multi-slit MOS capability the choice of a 7-hex fiber slicer would seem to offer the best intermediate dispersion $(R<20,000)$ compromise giving object multiplex gains of $\sim 140$ in each CHRS camera assuming a nod-andshuflle mode of observing.

\begin{tabular}{|c|c|c|c|c|}
\hline $\begin{array}{c}\text { SLICER } \\
(0.75 ”)\end{array}$ & $\begin{array}{c}\text { FIBER DIA. } \\
(\mu \mathrm{m})\end{array}$ & $\begin{array}{c}\mathrm{R}_{\text {Octave }} \\
\left(\lambda_{0} \sim 600 \mathrm{~nm}\right)\end{array}$ & $\begin{array}{c}\mathrm{R}_{1200} \\
(\Delta \lambda \sim 110 \mathrm{~nm})\end{array}$ & $\begin{array}{c}\mathbf{M}_{\#} \\
(2 \text {-arms+N\&S })\end{array}$ \\
\hline Single fiber & 600 & 1,400 & 5,400 & 700 \\
\hline 7-hex & 225 & 3,600 & 14,500 & 280 \\
\hline 19-hex & 140 & 5,400 & 18,500 & 170 \\
\hline 37-hex & 100 & 5,400 & 18,700 & 120 \\
\hline
\end{tabular}

Table 1: Examples of Fiber-mode Options

With sensitivity goals of $<5$ magnitude below sky all spectroscopic instruments are going to require strict control of systematic errors whereas fiber instruments, traditionally, are characterized by $\sim 2 \%$ skysubtraction errors. Fortunately the development of charge-shuffling ${ }^{7}$ in consort with aperture nodding (N\&S mode) have the potential to establish almost perfect, Poisson-noise limited, sky-subtraction and while it is true that for N\&S work $<50 \%$ of the detector real-estate and only $50 \%$ of the exposure time is allocated to on-source integration this is to be compared with the traditional long-slit MOS approach where factors of $>4$ in detector real-estate are dedicated to sky.

We have assumed here that a CELT fiber positioner would be based on that of $\mathrm{OzPoz}^{8}$, the Nasmyth positioner for the VLT FLAMES facility'. Apart from up-scaling the design, very little needs to be changed in the basic concept. Its 4 field-plates would allow for a variety of options including different sized fibers and fiber-based d-IFUs. Each field-plate could, in principle, support $\sim 1800$ fiber buttons, well in excess of the MTHR concept (even in its low dispersion, $M_{\#} \sim 700$ mode) and hence a variety of options could be supported including different fiber apertures, different slicing geometries non-N\&S (2* $\left.\mathrm{M}_{\#}\right)$ modes not to mention a range of deployable fiber-based IFUs (d-IFUs).

\subsection{Low Dispersion Multi-slit Spectrograph (CFOS)}


One clear science case requirement which is difficult to satisfy with MTHR is the important low dispersion, faint limit optical spectroscopy normally satisfied through wide-field multi-slit MOS devices. Here the difficulties are not only in the scale of the optics implied by the $20^{\prime}, \mathrm{f} / 15$ focus but also in the requirement for atmospheric dispersion compensation (ADC) over the full field. The chosen approach to this challenge is to look at the possibility of an $\mathrm{f} / 15$ to $\mathrm{f} / 5$ converter (plus ADC) feeding a 4-shooter spectrograph. A preliminary design is discussed.

The original concept for the focal reducer ${ }^{10}$ (the 3-mirror focal reducer or $3 \mathrm{MfR}$ ) was produced by Damien Jones (Prime Optics, Qld). The preliminary design folds the beam vertically using a large atoroidal field mirror at the native $2.6 \mathrm{~m}$ diameter $\mathrm{f} / 15$ focus. The beam is then re-imaged to a more manageable $\sim 870 \mathrm{~mm}$ focus by a triple mirror anastigmat (TMA) to produce an $\mathrm{f} / 5$ image surface whose distortion is stable to field rotation. There is sufficient space between the last element of the TMA and the input focal plane of the spectrograph to supply a classical Risley prism ADC whose diameter will be well under $\sim 1$ metre. The proposed arrangement is to have the spectrograph upward looking and rotating about a vertical axis ideal for such a massive instrument.

There are a large variety of possibilities for the spectrograph design, however the requirement to produce spectra over such a wide field falls easily into a VIMOS-like concept ${ }^{11}$ whereby the field is divided by 4 field lenses into contiguous quadrants. Each field-lens/collimator combination then creates its own pupil where the light is dispersed into 4 separate cameras. Such a format leads to individual $\sim 10^{\prime}$-by-7' fields of view for each spectrograph (a total rectangular field of 20'-by-14' with some vignetting losses in the extreme corners) which are imaged at $\sim \mathrm{f} / 1.1$ onto a $6 \mathrm{k}^{2}(15 \mu \mathrm{m}$ pixel) detector. At such speeds the cameras are, of necessity, internal focus Schmidt cameras (or their variants) but for such a beam size the vignetting of the detector package may be $\sim 10 \%$.

A $400 \mathrm{~mm}$ collimated beam combined with a mosaic of $1200 \mathrm{l} / \mathrm{mm}$ standard surface-ruled gratings mounted at a $\sim 40^{\circ}$ camera/collimator angle would then produce $1^{\text {st }}$ order $(\lambda \sim 600 \mathrm{~nm})$ resolutions $R \sim 3,600$ for slitwidths of 0.75 " over a $\sim 120 \mathrm{~nm} \lambda$-range; a full octave could be recovered at $R \sim 850$. However we suggest here the use of VPH gratings in a transmission Littrow configuration ${ }^{12}$ to give higher dispersions and greater blaze efficiencies. As a comparison a $2400 \mathrm{l} / \mathrm{mm}$ VPH so configured could give $\mathrm{R} \sim 7,600$. While the necessity for articulated cameras creates more mechanical complexity the efficiency and spectral resolution gains are substantial. Furthermore camera articulation has the added benefit of permitting direct imaging by simple removal of the grating leading to a spectacular 0.1 " sampled, 20-by-14' imaging field on CELT.

\section{SINGLE-CONJUGATE ADAPTIVE OPTICS (SCAO) INSTRUMENTS}

Wavelengths longer than $2.5 \mu \mathrm{m}$ are difficult to study from ground-based telescopes because of the thermal emission generated by the telescope optics and the Earth's atmosphere. Nevertheless, the exceptional angular resolution obtainable with CELT makes it desirable to observe proto-planetary disks that space missions cannot resolve. Recalling that the diffraction-limit of CELT is $\sim 70$ mas at $10 \mu \mathrm{m}$, then CELT could be diffraction-limited at these wavelengths with only modest wavefront corrections. At these wavelengths, it is expected that the kind of AO systems available today will be sufficient. The thermal background generated by the finite emissivity of the mirrors of the AO system (and telescope) however will be a drawback. Any mid-IR instrument will require either an optimal location (e.g. at a special focus preceded by an adaptive secondary) or a cryogenic $\mathrm{AO}$ system. In addition, the instrument will need to contain a cryogenic pupil stop that can be rotated and synchronized to the motion of the telescope pupil.

The primary instrument envisaged for the SCAO mode is a mid-IR camera/spectrometer combination covering the wavelength range from $8-27 \mu \mathrm{m}$, but with performance optimized for the $8-13 \mu \mathrm{m}$ window.

Key requirements of the design would be:

- A Coronagraphic mode with occulting disk and rotating Lyot stop

- Grism spectroscopy with $\mathrm{R}>4,000$ 
- Nyquist sampling - the broad wavelength range requires two cameras

- $34 \mathrm{mas} / \mathrm{pixel}$ at $10 \mu \mathrm{m} ; 69 \mathrm{mas} / \mathrm{pixel}$ at $20 \mu \mathrm{m}$

- Final f-ratio: $\mathrm{f} / 5.4$

- $\quad 70$ " field of view with $2 \mathrm{k}^{2}$ As:Si array

Detectors of this size are not currently available for this wavelength region, and they would be challenging to produce for applications other than spectroscopy because of the number of readout channels and very high speeds required.

The requirement for a broad wavelength range and high throughput suggests an all-reflecting, non-obscured optical design. The $f / 15$ collimator will have a $\sim 3.8^{0}$ field of view while the $f / 5.4$ camera has $\sim 10.6^{0}$. This combination of $\mathrm{f}$-number and field of view implies that simple configurations such as a pair of off-axis parabolas will not provide adequate control of aberrations for diffraction limited performance. Both optical elements probably need to be three-mirror anastigmats (TMAs) but a detailed design is not yet available.

The best detectors for this range are Si:As BIB devices. It is assumed that large formats will be developed for NGST and other applications. In estimating performance and design, the following detector properties were assumed. A $2 \mathrm{k}^{2}$ As:Si detector with a pixel pitch of $27 \mu \mathrm{m}$ and no gaps (total detector size of $55.3 \times 55.3 \mathrm{~mm}$ ) and an operating temperature of $8^{0} \mathrm{~K}$. The dark current is assumed to be $1 \mathrm{e}-/ \mathrm{s}$, the readout noise is $4 \mathrm{e}-\mathrm{rms}$ and the charge storage capacity or well depth is $3.10^{7} \mathrm{e}-$.

\section{MULTI-CONJUGATE ADAPTIVE OPTIC (MCAO) INSTRUMENTS}

This mode will primarily be optimized for the near-IR $(1-2.5 \mu \mathrm{m})$. with a corrected field specified to no more than $\sim 2$ '. While a direct camera for normal imaging at the diffraction limit of the telescope is desirable, since the diffraction limit is $\sim 7$ mas at $1 \mu \mathrm{m}$, it would require $2 \mathrm{k}$ pixels to Nyquist sample a field of only 7". Therefore, rather than propose a massive imager we have integrated an imaging capability into plans for a deployable (d-)IFU spectrograph.

For spatially resolved IFU spectroscopy, fundamental instrument parameters depend critically on the science goals. Given typical core sizes ( $\sim 0.3$ ") and surface brightnesses of $z>1$ galaxies, spatially resolved spectroscopy at the CELT diffraction limit will generally result in marginal signal-to-noise ${ }^{3}$. We assume, therefore, modest spatial samplings in the region 25-100mas for this work. Furthermore, at K 22.5 the field galaxy surface density is $\sim 20 / \mathrm{sq}$.arcmin. implying $\sim 60$ deployable d-IFUs within the 2 ' diameter MCAO field. While with sub-galactic masses expected for the majority of such objects, spectral resolutions above $R \sim 3000$ are required to gain kinematic information, $R \sim 4000$ is perhaps a better value for optimal OHsuppression. On the other hand, a variety of science targets such as solar system objects, proto-stellar disks, quasar hosts, the Galactic center, and nearby AGN also benefit from integral field spectroscopy but do not require multi-object capabilities. These may be better suited to a "dense-pack", large-IFU configuration that can, in principle, use the same d-IFU spectrograph, however, the implementation is critically dependant on chosen technology and will not be discussed further here.

As an example, we baseline 16 IFUs (d-IFUs) with 50mas spatial sampling deployed over a 2' diameter, MCAO, field. Each IFU subtends a 1.4"-by-1.7" field and feeds 2k spectro-spatial X-pixels and 1k spatial Y-pixels. Given this significant investment in detector real estate (8-by- $2 \mathrm{k}^{2} \mathrm{HgCdTe}$ arrays) it seems prudent to investigate ways of using these for MCAO-fed imaging. A near-diffraction-limited spatial resolution of $\sim 5 \mathrm{mas} / \mathrm{pixel}$ was chosen leading to the prospect of 16 deployable imager units (or d-IUs) each having a $\sim 5$-by-10" field. The slicer concept and the deployment scheme is based on the UIST instrument ${ }^{13}$.

There are 3 identifiable optical sub-systems between the MCAO output focal-plane and the spectrograph input slit. 
1. The pick-off Arm probe (PoA) relays the native $\mathrm{f} / 15$ image through a cold stop to a secondary $\sim \mathrm{f} / 30$ image. Through the use of a selector mirror, this secondary, magnified image can be directed either to: the fore-optics to the d-IFU system, or the re-imaging path of the d-IU system.

2. In entering the $d-I F U, a$ three mirror toroidal relay takes the secondary, $\mathrm{f} / 30$ image and converts it into an $\sim \mathrm{f} / 100$ image formed on the surface of the slicer thus matching its 50 mas sampling to the $\sim 1 \mathrm{~mm}$ physical thickness of each slice, chosen for ease of manufacture. The factor of $\sim 2$ induced anamorphism implies that the Y-direction of the image is formed at $\sim \mathrm{f} / 50$.

3. The 28 tilted slices of the IFU are each $\sim 25 \mathrm{~mm}$ in length and are spherically profiled to form a linear array of 28 separate pupil images. Each individual pseudo-slitlet is then relayed through pupil mirrors to form a linear stack of slitlets with a total height of $\sim 74 \mathrm{~mm}$.

Each of 4 spectrographs services 4 d-IFU PoAs (16 PoAs in total). Now each $74 \mathrm{~mm}$ long, pseudo-slit produces an $\mathrm{f} / 20$ beam exiting in the spectro-spatial dispersion (or $\mathrm{X}$-) direction and an $\mathrm{f} / 10$ beam in the spatial (or Y-) direction. We choose a fully transmissive, grism-based spectrograph for conceptual simplicity although a full optical and engineering design evaluation may well reveal a more compact or efficient arrangement. A collimator focal-length of $\sim 2 \mathrm{~m}$ is assumed to give adequate, $R \sim 4000$, spectral resolution while limiting the field-angles into the collimator. A camera $\mathrm{f}$-ratio of $\mathrm{f} / 5$ (X-direction) will thus produce an image of the psuedo-slit on the detector matched to $1 \mathrm{k}$ of its $18 \mu \mathrm{m}$ pixels. In this way an arrangement of $4 \mathrm{~d}$-IFU psuedo-slits can be supported by a single spectrograph having a $2 \mathrm{k}$-by-4k format formed from two close butted $\mathrm{HgCdTe}$ arrays.

The relatively slow collimators and cameras would have modest field-angles and hence their optical design should not be too challenging. Each spectrograph is likely to be over $3.5 \mathrm{~m}$ in length which may well argue for additional folds in the collimated beam, as was suggested in the GIRMOS study ${ }^{14}$. Without such folds the 4 spectrograph could be arranged $90^{\circ}$ apart in a $\sim 1 \mathrm{~m}$ radius ring and hence the whole d-IFU instrument from PoA to spectrograph would be contained in a $\sim 3.5 \mathrm{~m}$ long, $\sim 2.5 \mathrm{~m}$ diameter cylindrical dewar.

Through the use of the selector mirror, the secondary $\mathrm{f} / 30$ image formed at the output of each PoA can bipass the d-IFU unit and be relayed, via a selectable roof prism, directly onto the detector itself. A scale of 5 mas per pixel implies an $\sim \mathrm{f} / 25$ re-imager incorporating an intermediate cold stop for the placement of suitable filters. Again, 4 PoAs would be able to service a single $4 \mathrm{k}$-by- $2 \mathrm{k}$ array of 2 Hawaii-2RGs giving each a 10"-by-5" field of view and hence all 16 PoAs could be used for non-contiguous MCAO imaging.

\section{EXTREME AO (EXAO) INSTRUMENTS}

Even in a "perfect" image of a star, the stellar light is distributed in the diffraction pattern of the telescope, not a concentrated point source. Coronagraphic techniques can be used to suppress telescope diffraction and render the image plane darker near the central star. In its simplest form, the Lyot coronagraph, a focal stop blocks a large fraction of the light by occulting the central few $\lambda / \mathrm{D}$ of the image. Light that is diffracted to a larger angle by passing close to a diffracting element (pupil edge, spider, or segment gap) passes the focal stop, but can be re-imaged in a following pupil plane. In the pupil plane, this light is closely associated with the edges that caused the diffraction, and can be blocked with a Lyot (undersized pupil) stop. A trade must be made between the size of the occulting spot and the fraction of light blocked. For a $4 \lambda / \mathrm{D}$ diameter stop, $50 \%$ of the pupil is blocked. At high strehl, apodisation of the focal stop with a smoothly varying transmission profile can significantly improve rejection of on-axis light. The Lyot coronagraph is robust and straightforward to implement, but suffers the disadvantages of limited rejection, low throughput, and completely obscuring the central field of view. Nulling and interferometric coronagraph techniques can circumvent some of these limitations. Particularly promising is the phase quadrant coronagraph, which rejects all on-axis light from a perfect unobscured aperture. Its principal disadvantage is an extreme sensitivity to residual tip/tilt errors. The on-axis light appears outside the image of the telescope pupil. If the pupil mask is a mirror with a hole, the on-axis starlight can be refocused to a high bandwidth tip/tilt control loop to ensure sufficient centering on the phase mask. Such a coronagraph 
behind an ExAO system would reject nearly all of the light in the diffraction pattern of the telescope. These novel coronagraphic techniques are presently being proven on existing high order $\mathrm{AO}$ systems.

After suppression of diffraction by a coronagraph, a science instrument focused on the science goals of the ExAO system is required. For the primary science driver, direct imaging of extrasolar planets, different approaches are likely depending on whether the position of the planet is unknown or known (eg. via astrometric orbits from SIM or Keck interferometer). If the position of the planet is known, then it is not necessary to have a large number of spatial pixels. In this case, it would be possible to apply techniques to further suppress the light in the PSF and speckle pattern by taking advantage of properties of the planet (eg methane absorption, polarization), properties of the speckle pattern (eg wavelength dependant scaling, dark speckles) or both with an integral field spectrograph, simultaneous dual imaging polarimeter, or photon counting dark speckle camera.

\section{GROUND-LAYER AO (GLAO) INSTRUMENTS}

A new, but potentially exciting, possibility is that offered by so-called Ground-Layer AO (GLAO). This is an $\mathrm{AO}$ technique which corrects only for the lowest turbulent atmospheric layer in order to deliver significantly improved "seeing" over a large $\sim 5$ ' field of view. As such it represents an important crossover technique that makes no attempt to deliver diffraction-limited imagery but nevertheless corrects for much of the spatially correlated, low frequency power of the atmosphere (and telescope). For spectroscopy, where input aperture coupling and field of view are often at more of a premium than observing at the diffraction limit GLAO promises very substantial gains.

GLAO is still very much at the conceptual stage and so we can only make informed guesses as to its performance. For the sake of argument, we will assume that CELT will have a GLAO facility which will deliver a factor of $\sim 2$, or more, improvement in "seeing" for $\lambda>0.8 \mu \mathrm{m}$ over a $\sim 4$ ' diameter field. Were this to be the case then a fairly simple adaptation of current near-IR spectrograph designs from Keck to CELT could be envisaged. As an example, we migrate the proposed KIRMOS design ${ }^{15}$ to make it compatible with a CELT GLAO facility.

KIRMOS, as proposed, is a near-IR, wide-field $\mathrm{f} / 15$ to $\mathrm{f} / 2.3$ all transmissive focal reducer for use as an MOS spectrograph/imager for Keck. It supplies a $4 \mathrm{k}^{2} \mathrm{HgCdTe}$ array made from 4 edge-butted $2 \mathrm{k}^{2}$ arrays giving it a huge 11'-by-11' field of view while the $175 \mathrm{~mm}$ beam permits $\mathrm{R} \sim 4,000 \mathrm{~J}, \mathrm{H}, \mathrm{K}$ (grism) spectroscopy when matched to a 0.75 " slit. A very similar CELT GLAO instrument would require a relatively small perturbation to the KIRMOS optical prescription to give it a $\sim 3.5$ '-by-3.5' field. In this case a very similar optical system would produce $\mathrm{R} \sim 4000 \mathrm{~J}, \mathrm{H}, \mathrm{K}$ spectroscopy for 0.25 " slits. In principle object multiplexes of $>100$ could be achieved with a traditional multi-longslit approach while nod-andshuffle micro-holes could increase this by more than a factor of 2 .

\section{ACKNOWLEDGEMENTS}

We would like to thank all our colleagues who contributed to the CELT Phase A design study and in particular the members of the CELT Instrumentation Working Group (Puragra Guhathakurta, James Graham, James Larkin and Steve Vogt) who contributed much material on which this summary paper is based. KT would also like to acknowledge the assistance of Bob Weber and Sean Lin (Caltech) who created the bulk of the engineering designs for the study; optical design consultant Damien Jones (Prime Optics, Qld., Australia) and Sam Barden (NOAO) who freely shared his own ideas during the parallel GSMT study.

\section{REFERENCES}

1. Scientific Opportunities with 30m Ground-Based Telescopes (Ellis, 2002) [SPIE: 4841] 
2. Taylor et al (1996): The AUSTRALIS Study Report (http://msowww.anu.edu.au/ colless/AUSTRALIS/)

3. CELT Technical Note 12: System Modeling (Baldry and Taylor, 2001) (http://celt.ucolick.org/reports_notes.html)

4. CELT Technical Note 19: The MTHR Spectrometer: Conceptual Design Study. Steven S. Vogt (2001) (http://celt.ucolick.org/reports notes.html)

5. Kenworthy, Parry and Taylor K. (2001) "SPIRAL Phase A: A Prototype Integral Field Spectrograph for the AAT" PASP 113, p215

6. Glazebrook \& Bland-Hawthorn (2001) "Microslit Nod-Shuffle Spectroscopy" PASP, 113, 197

7. Bland-Hawthorn (1996), Anglo-Australian Newsletters 79, p14

8. Performance of OzPoz, a multi-fiber positioner on the VLT: P. R. Gillingham, D. Popovic, L. G. Waller, T. J. Farrell, [SPIE: 4841-126]

9. Commissioning and astronomical performances of FLAMES, the VLT multifiber facility: L. Pasquini et al. [SPIE 4841-187]

10. CELT Technical Note 19: 3MfR (Jones and Taylor, 2001) (http://celt.ucolick.org/reports notes.html)

11. Performances of the VLT-VIMOS instrument: O. LeFevre et al. [SPIE: 4841-188]

12. Robertson et al (2000) ATLAS: a Cassegrain spectrograph based on volume phase holographic gratings: Proc. SPIE: 4008, p194

13. Wells et al (2000) Design and testing of a cryogenic image slicing IFU for UKIRT and NGST: Proc. SPIE: 4008, p1215

14. Wright et al (2000) GIRMOS: an infrared multi-object spectrograph for Gemini Proc. SPIE, 4008 , p729

15. KIRMOS Phase A Design Study (Taylor et al. 2002) Caltech Optical Observatories (Internal Report) 